\title{
Succinate Dehydrogenase Subunit B (SDHB) Is Expressed in Neurofibro- matosis 1-Associated Gastrointestinal Stromal Tumors (Gists): Implications for the SDHB Expression Based Classification of Gists
}

\author{
Jeanny H. Wang, Jerzy Lasota, and Markku Miettinen ${ }^{凶}$ \\ Department of Soft Tissue Pathology, Armed Forces Institute of Pathology, Washington, DC, USA
}

Corresponding author: Markku Miettinen, MD, Department of Soft Tissue Pathology, Armed Forces Institute of Pathology, 6825 16 th Street, NW, Washington DC 20306. Phone: 202-782-1575; Fax: 202-782-9182, E-mail: miettinen@afip.osd

(C) Ivyspring International Publisher. This is an open-access article distributed under the terms of the Creative Commons License (http://creativecommons.org/ licenses/by-nc-nd/3.0/). Reproduction is permitted for personal, noncommercial use, provided that the article is in whole, unmodified, and properly cited.

Received: 2011.01.22; Accepted: 2011.02.16; Published: 2011.02.16

\begin{abstract}
Gastrointestinal Stromal Tumor (GIST) is the most common mesenchymal tumor of the digestive tract. GISTs develop with relatively high incidence in patients with Neurofibromatosis-I syndrome (NFI). Mutational activation of KIT or PDGFRA is believed to be a driving force in the pathogenesis of familial and sporadic GISTs. Unlike those tumors, $\mathrm{NFI}$-associated GISTs do not have KIT or PGDFRA mutations. Similarly, no mutational activation of KIT or PDGFRA has been identified in pediatric GISTs and in GISTs associated with Carney Triad and Carney-Stratakis Syndrome. KIT and PDGFRA-wild type tumors are expected to have lesser response to imatinib treatment. Recently, Carney Triad and Carney-Stratakis Syndrome -associated GISTs and pediatric GISTs have been shown to have a loss of expression of succinate dehydrogenase subunit B (SDHB), a Krebs cycle/electron transport chain interface protein. It was proposed that GISTs can be divided into SDHBpositive (type I), and SDHB-negative (type 2) tumors because of similarities in clinical features and response to imatinib treatment. In this study, SDHB expression was examined immunohistochemically in 22 well-characterized NFI-associated GISTs. All analyzed tumors expressed SDHB. Based on SDHB-expression status, NFI-associated GISTs belong to type I category; however, similarly to SDHB type 2 tumors, they do not respond well to imatinib treatment. Therefore, a simple categorization of GISTs into SDHB-positive and-negative seems to be incomplete. A classification based on both SDHB expression status and KIT and PDGFRA mutation status characterize GISTs more accurately and allow subdivision of SDHB-positive tumors into different clinico-genetic categories.
\end{abstract}

Key words: GIST, Neurofibromatosis-1, SDHB, Carney Triad, Carney-Stratakis Syndrome, KIT, PDGFRA, mutation, imatinib

\section{Introduction}

Neurofibromatosis-1 (NF1), also known as von Recklinghausen disease, is an inherited, autosomal dominant disease phenotypically characterized by multiple café au lait spots, Lisch nodules, freckling, and development of neurofibromas, and in some cases, their malignant derivatives [1]. Also, gastrointes- tinal stromal tumors (GISTs) arise in NF1 patients 150 times more frequently than in the general population [2]. With an estimated birth incidence of 1:3000 in Western countries, NF1 is the most commonly inherited disease [1]. It was postulated that NF1 is caused by a loss-of-function of tumor suppressor gene lead- 
ing to the inactivation of neurofibromin that regulates cell proliferation and cell differentiation through inhibitory regulation of the ras oncogene, a GTPase [3-7].

Gastrointestinal stromal tumor (GIST) is the most common mesenchymal neoplasm of the gastrointestinal tract. GISTs are believed to originate from the interstitial cells of Cajal, pacemaker cells that regulate peristalsis in the digestive tract [8]. An oncogenic (mutational) activation of KIT or PDGFRA tyrosine kinases has been identified in a great majority of GISTs [9]. However, no GIST-specific KIT or PDGFRA mutations were identified in GIST associated with NF1, Carney Triad (CT), Carney-Stratakis Syndrome (CSS), in pediatric GISTs, or in a small subset $(<10 \%)$ of sporadic adult GISTs [10-14]. Molecular mechanisms underlying the development of KIT/PDGFRA-wild type (WT) GISTs are not well understood.

Recently, GISTs associated with CT (triad of gastric GIST, pulmonary chondroma, and extra-adrenal paraganglioma) and CSS (hereditary GIST paraganglioma syndrome) have been shown to have a loss of succinate dehydrogenase subunit $\mathrm{B}$ (SDHB) expression $[15,16]$. SDHB, a mitochondrial protein, is one of the ubiquitously expressed proteins functioning at the interface of tricarboxylic acid cycle and electron transport chain. It is believed that loss of SDHB expression leads to a tumorigennic mechanism involving the stabilization of Hypoxia Inducing Factors (HIF), which in turn upregulates genes that promote adaptation of cells in low-oxygen environments by inducing angiogenesis and glycolysis [17-20].

Data on SDHB expression in NF1-associated GISTs are limited and only three cases were tested and turned to be SDHB- positive [15]. The aim of this study was to evaluate SDHB expression in a significant number of NF1-associated GISTs and test a hypothesis that a lack of SDHB expression, unlike in CTand CSS-associated tumors and pediatric GISTs, is not involved in pathogenesis of NF1-associated GISTs.

\section{Materials and Methods}

A total of 23 NF1-associated GISTs, from years 1970-1999, were obtained from the Armed Forces Institute of Pathology (AFIP) tissue repository.

Immunostaining was performed with Leica Bond-Max automatic immunostainer (Leica, Bannockburn, IL) using a commercially available SDHB mouse monoclonal antibody 21A11 (Abcam ab14714, Cambridge, MA). Heat induced epitope retrieval (high $\mathrm{pH}$, EDTA-based buffer, $\mathrm{pH}$ 9.0, Leica Bond-Max) was applied for 25 minutes. The primary antibody was incubated for 30 minutes at room temperature. Leica Bond-Max avidin-biotin free polymer system was used in the detection according to the recommended procedure. Diaminobenzidine was used as the chromogen, following the blocking of endogenous peroxidase with $3 \%$ hydrogen peroxide diluted in phosphate buffer. Immunostained slides were counterstained with hematoxylin. Only stains with positive internal controls (granular cytoplasmic staining in vascular endothelia and pericytes) were considered valid. One case with unsuccesful immunostaining (internal control negative) was removed from the study.

\section{Results}

All twenty two NF1-associated GISTs exhibited granular, cytoplasmic staining for SDHB (Fig. 1). In the analyzed cohort, there were $8(36.4 \%)$ male and 14 $(63.6 \%)$ female patients. Median age at tumor diagnosis was 41 years (mean, 46 years). Twenty one tumors were located in the small intestine and one in colon. Tumor sizes varied from 0.9 to $10 \mathrm{~cm}$ (median, 3.5 $\mathrm{cm}$ ), and 15 patients presented with multiple tumors. Most of these GISTs had mitotic counts $<5$ per 50 HPFs, and only two tumors contained more than 5 mitoses per 50 HPFs. KIT and DOG 1/Anoctamin-1 were expressed in all tumors. GIST-specific KIT exon 9, 11, 13, and 17, and PDGFRA exon 12, 14, and 18 mutations were not found as previously reported [11].

\section{Discussion}

In this study, we demonstrate consistent SDHB expression in neurofibromatosis 1 (NF1)-associated GISTs. Clinicopathologic features of these GISTs include patient female predominance, small intestinal location, common multiplicity, KIT and DOG 1 expression, and lack of GIST-specific KIT and PDGFRA mutations, as previously reported [10, 11]. Lack of GIST-specific KIT and PDGFRA mutations has also been reported in pediatric GISTs, Carney triad (CT) and Carney-Stratakis (CS) syndrome-associated GISTs, and in a small subset of adult GISTs [10-14].

Lack of SDHB expression has been reported in Carney triad (CT) and Carney-Stratakis (CS) syndrome-associated GISTs and more recently in pediatric and adult sporadic GISTs showing morphologic and clinical features similar to CT- and CS-GISTs. It has been proposed that based on SDHB expression, GISTs could be divided into 2 distinctive subtypes: SDHB- positive (type 1) and SDHB-negative (type 2) tumors [15]. Type 1 GISTs can occur in adults anywhere in the gastrointestinal tract, have equal sex distribution, generally have KIT or PDGFRA mutations, and, in majority, respond to imatinib treatment. 
Type 2 GISTs occur in childhood or young adulthood nearly exclusively in the stomach, show a striking predilection for the female sex and epitheliod morphology. These tumors lack KIT or PDGFRA mutations and show a limited response to imatinb inhibition [14].

Based on SDHB-expression status, NF1-associated GISTs belong to type 1 category. However, they differ from type 1 GIST characteristics by restriction of occurrence to intestines, common tumor multiplicity, female predominance, and consistent lack of GIST-specific KIT and PDGRA mutations. Similar to SDHB type 2 tumors, they do not respond well to imatinib treatment [21-23]. Therefore, clinicopathologically NF1-associated GISTs deserve a separation from other type 1 (SDHB-positive) GISTs.

Although imatinib mesylate, KIT and PDGFRA tyrosine kinase inhibitor was successfully used for treatment of advanced and disseminated GISTs [24], such a treatment is ineffective or minimally effective in KIT/PDGFRA-wild type GISTs, as well as those with certain mutations, such as PDGFRA Asp842Val substitution [25]. Thus, genotyping of GISTs is considered a standard clinical test in all disseminated GISTs and tumors with a substantial risk of developing metastatic disease.

In conclusion, NF1-associated GISTs express SDHB, similar to a majority of adult GISTs. In view of different KIT/PDGFRA mutation status and prospect to targeted treatment, simple categorization of GISTs into type 1 and type 2 based on SDHB expression is inadequate. A classification based on both SDHB expression status and other clinicopatholopgic factors: KIT/PDGFRA mutation and NF1 syndrome status characterize GISTs more accurately allowing subdivision of SDHB-positive tumors into different categories based on these clinicopathologic factors (Table 1).

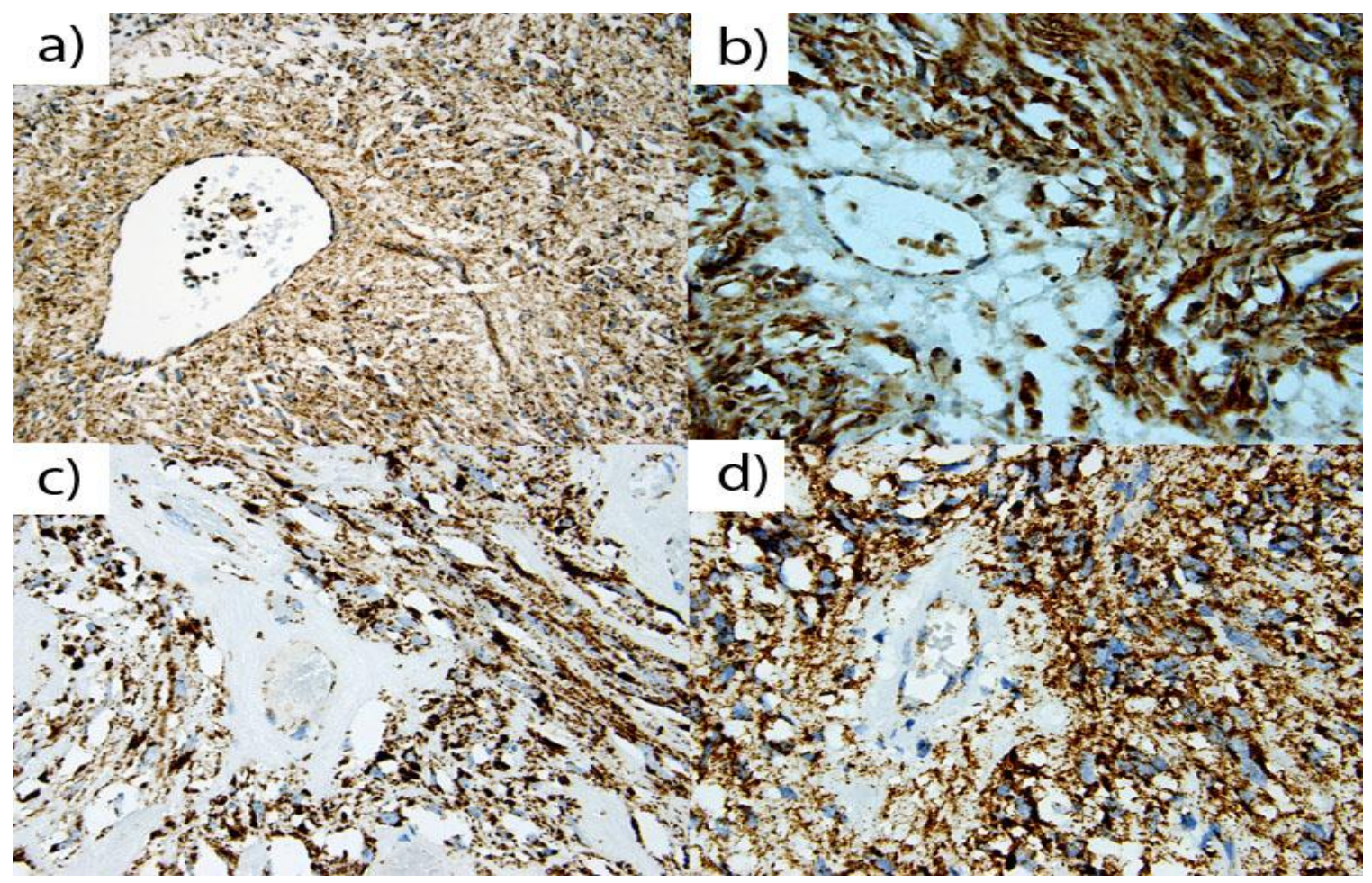

Figure I: A representative of Nf-I GISTs of (a) 67 year old male, (b) 36 year old female, (c) 25 year old male, and (d) 60 year old male. A majority of Nf-I associated GISTs exhibited positive, granular, cytoplasm staining for SDHB in gastrointestinal stromal tumors. All positively stained cells show granular cytoplasm (mitochondrial) staining pattern. 
Table I. Classification of GISTs based on SDHB expression and KIT or PDGFRA mutation status.

\begin{tabular}{ccc}
\hline SDHB status & KIT/PDGFRA mutation & Clinical features \\
\hline SDHB-positive & $\begin{array}{c}\text { KIT exon 9, 11, 13, and 17; } \\
\text { PDGFRA exon 12, 14, and 18 }\end{array}$ & $\begin{array}{c}\text { Sporadic or familial tumors (adult type) Generally high, but varies (lower for KIT exon 9 } \\
\text { mutants and poor response for the most com- } \\
\text { mon PDGFRA exon 18 mutant (D842V) }\end{array}$ \\
\cline { 2 - 3 } & KIT/PDGFRA-WT & Sporadic \\
KIT/PDGFRA-WT & Neurofibromatosis-1 Syndrome \\
\hline SDHB-negative & Low & Carney Triad \\
\hline
\end{tabular}

\section{Conflict of Interest}

The authors have declared that no conflict of interest exists.

The opinions and assertions contained herein represent the personal views of the authors and are not to be construed as official or as representing the views of the Department of the Army or the Department of Defense.

\section{References}

1. Ward BA and Gutmann DH. Neurofibromatosis 1: from lab bench to clinic. Pediatr Neurol. 2005;32:221-228.

2. Bajor J. Gastrointestinal stromal tumours in patients with type 1 neurofibromatosis. Clin Exp Med J. 2009;3:247-254.

3. Wallace MR, Marchuck DA, Anderson LB, et al. Type 1 neurofibromatosis gene: identification of a large transcript disrupted in three NF1 patients. Science. 1990;249: 181-186.

4. Carroll SL and Stonecypher MS. Tumor suppressor mutations and growth factor signaling in the pathogenesis of NF1-associated peripheral nerve sheath tumors. I. The role of tumor suppressor mutations. J Neuropathol Ex Neurol. 2004;63:1115-1123.

5. Carroll SL, Stonecypher MS. Tumor suppressor mutations and growth factor signaling in the pathogenesis of NF1-associated peripheral nerve sheath tumors: The role of dysregulated growth factor signaling. J Neuropathol Ex Neurol. $2005 ; 64: 1-9$.

6. Reed N, Gutmann DH. Tumorigenesis in neurofibromatosis: new insights and potential therapies. Trends Mol Med. 2001;7:157-162.

7. Yohay KH. The genetic and molecular pathogenesis of NF1 and NF2. Semin Pediatr Neurol. 2006;13:21-26.

8. Miettinen M, Lasota J: Pathology and prognosis of gastrointestinal stromal tumors at different sites. Semin in Diagn Pathol. 2006;23:70-83

9. Lasota J, Miettinen M. Clinical significance of oncogenic KIT and PDGFRA mutations in gastrointestinal stromal tumours. Histopathology. 2008;53:245-266

10. Andersson J, Sihto H, Mies-Kindbloom JM, et al. NF1- associated gastrointestinal stromal tumors have unique clinical, phenotypic, and genotypic characteristics. Am J Surg Pathol. 2005;29:1170-1176.

11. Miettinen M, Fetsch JF, Lasota J, et al. Gastrointestinal stromal tumors in patients with neurofibromatosis 1: a clinicopathologic and molecular genetic study of 45 cases. Am J Surg Pathol. 2006;30:90-96.
12. Miettinen M, Lasota J, Sobin LH. Gastrointestinal stromal tumors (GISTs) of the stomach in children and young adults: a linicopathologic, immunohistochemical and molecular genetic study of 44 cases with long term follow-up and review of the literature. Am J Surg Pathol. 2005;29:1373-1381.

13. Price VE, Zielenska M, Chilton-MacNeill S, et al. Clinical and molecular characteristics of pediatric gastrointestinal stromal tumors (GISTs). Pediatr Blood Cancer. 2005;45:20-24.

14. Pasini B, McWhinney SR, Bei Th, at al. Clinical and molecular genetics of patients with the Carney-Stratakis syndrome and germline mutations of the genes coding for the succinate dehydrogenase subunits SDHB, SDHC, and SDHD. Eur J Hum Genet. 2008;16:79-88.

15. Gill AJ, Chou A, Vilain R et al. Immunohistochemistry for SDHB divides gastrointestinal stromal tumors (GISTs) into 2 distinctive types. Am J Surg Pathol. 2010;34:636-644.

16. Gaal J, Stratakis CA, Carney JA. SDHB immunohistochemistry: a useful tool in the diagnosis of Carney-Stratakis and Carney triad gastrointestinal stromal tumors. Mod Pathol. 2011 Jan;24(1):147-51.

17. Eng C, Kiuru M, Fernandez MJ et al. A role for mitochondrial enzymes in inherited neoplasia and beyond. Nat Rev Cancer. 2003;3:193-202.

18. Rustin P, Munnich A, Rotig A. Succinate Dehydrogenase and Human diseases: new insights into a well-known enzyme. Eur J Hum Genet 2002;10:289-291.

19. Selak MA, Armour SM, Mackenzie ED, et al. Succinate links TCA cycle dysfunction to oncogenesis by inhibiting HIF-alpha prolyl hydroxylase. Cancer Cell. 2005;7:77-85.

20. Pollard PJ, Wortham NC and Tomlinson IP. The TCA cycle and tumorigenesis: The examples of fumarate hydratase and succinate dehydrogenase. Ann Med. 2003;35:632-639.

21. Mussi C, Schildhaus HU, Gronchi A, et al. Therapeutic consequences from molecular biology for gastrointestinal stromal tumor patients affected by neurofibromatosis type 1 . Clin Cancer Res. 2008;14:4550-4555.

22. Lee JL, Kim JY, Ryu MH, et al. Response to imatinib in KIT- and PDGFRA-wild type gastrointestinal stromal associated with neurofibromatosis type 1. Dig Dis Sci. 2006;51:1043-1046.

23. Kalender ME, Sevinc A, Tutar E, et al. Effect of sunitinib on metastatic gastrointestinal stromal tumor in patients with neurofibromatosis type 1: a case report. World J Gastroenterol. 2007;13:2629-2632.

24. Joensuu H, Roberts PJ, Sarlomo-Rikala M et al. Effect of tyrosine kinase inhibitor STI571 in a patient with a metastatic gastrointestinal stromal tumor. N Engl J Med 2001; 344:1052-1056.

25. Heinrich MC, Corless CL, Blanke CD, et al. Molecular correlates of imatinib resistance in gastrointestinal stromal tumors. J Clin Onco 2006; 24: 4764-4774. 